\title{
Immunoglobulin concentrations in children receiving treatment for acute lymphoblastic leukaemia*
}

\author{
MM REID, $\dagger$ AW CRAFT, $\ddagger$ JR COX $\dagger$ \\ From the Departments of Haematology $\dagger$ and Child Health, $\ddagger$ Royal Victoria Infirmary, Newcastle upon Tyne
}

SUMMARY As part of a wider survey of infections and defence mechanisms, concentrations of serum immunoglobulins IgG, IgA, and IgM were measured regularly by single radial immunodiffusion in a group of children receiving treatment for acute lymphoblastic leukaemia while in their first remission. IgM concentrations were often markedly raised at diagnosis, and $\operatorname{IgG}$ and $\operatorname{IgA}$ concentrations both began falling within one month of diagnosis. Four months after diagnosis all three had fallen significantly, but this tendency stopped after six months. Only IgG partially recovered, and none returned to pretreatment concentrations even in children followed for two years. The results suggest that vincristine and prednisone are the major factors that initiated the fall in IgG and IgA. Lowered IgM concentrations may have been due to cranial irradiation or continuous maintenance treatment, or both.

The high risk of infection in children being treated for acute lymphoblastic leukaemia (ALL) has stimulated much research into their defence mechanisms. As part of a wider survey of infections and defence mechanisms in such children in Newcastle upon Tyne, immunoglobulins IgG, IgA, and IgM were measured regularly. Several studies of immunoglobulin concentrations in children with ALL have been reported, but there are major contradictions both in reports of concentrations at diagnosis and in the effects of treatment on them. Some of these differences may be due to the considerable variations in the treatment given. This study attempted to relate any changes in immunoglobulin concentrations to the different phases of treatment and was restricted to children receiving treatment who were in their first remission.

\section{Patients and methods}

From May 1975 to October 1976, 28 children with ALL were entered into the study. There were 13 boys and 15 girls. Their ages at diagnosis ranged from one to 10 years. All children received a modified version of the best arm of the Acute Leukaemia Group B

*This study was done on behalf of the Newcastle Leukaemia Research Group: C Barnaby, E Bruce, AW Craft, PS Gardner, J Kernahan, J McQuillin, TC Noble, RH Prudhoe, CR Pullan, MM Reid, PRA Taylor, W Walker.

Accepted for publication 6 October 1980
6801 protocol, details of which have been published elsewhere. ${ }^{1}$ The children were followed while in their first remission for periods ranging from two weeks to 24 months. Blood was taken at diagnosis and at monthly intervals when possible. It was not always possible to obtain enough blood to measure immunoglobulin concentrations in addition to the other demands of the survey. Between 15 and 19 children were assessed each month in the first six months and between 6 and 13 in the second six months of the follow-up period. Thereafter the number of children fell further.

The serum was separated, stored at $-20^{\circ} \mathrm{C}$, and measured in batches. IgG, IgA, and IgM were measured by single radial immunodiffusion on Diffu-Gel plates (Oxford) with the maximal diffusion method. Reference ranges were not established locally. Normal ranges have been established frequently by other workers using similar techniques, and for comparison those of Stiehm and Fudenberg ${ }^{2}$ have been used. Statistical analysis was done by Student's $t$ test.

\section{Results}

Mean concentrations ( \pm SD) of $\operatorname{IgG}, \operatorname{IgA}$, and IgM at diagnosis and at monthly intervals are shown in Figs 1, 2, and 3. At diagnosis IgG and IgA were similar to the reference ranges, but many children had high IgM with individual results well above normal. There was a tendency for all three classes of 


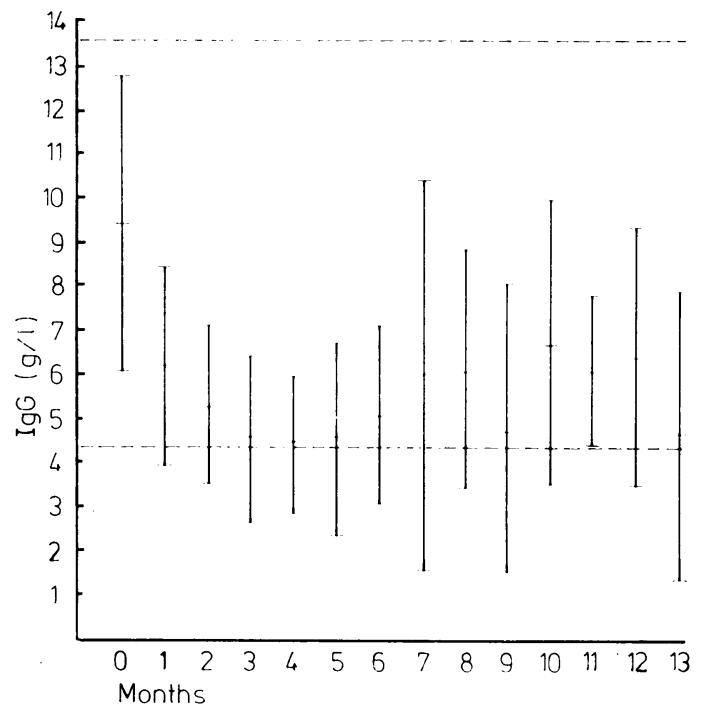

1

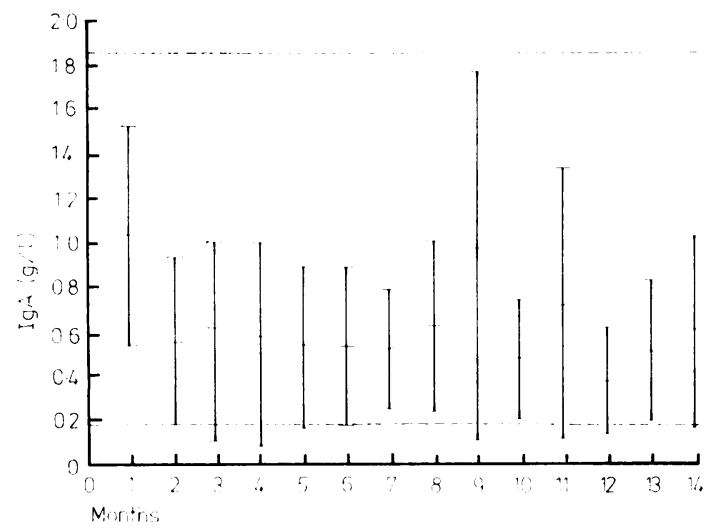

2

immunoglobulins to fall after this and it was most pronounced for IgG. The mean IgG one month after diagnosis fell significantly ( $<<0.005$ ) when compared to the pretreatment concentrations. Four months after diagnosis this fall was even more marked $(p<0.001)$. IgA also fell within one month of diagnosis $(p<0.005)$ but was not any lower four months after diagnosis. IgM, on the other hand, was not significantly different one month after diagnosis. By four months it too had fallen significantly $(p<0.0025)$. Mean levels are given for only 13 months. The longest period of follow-up was 24 months, but the number of regular monthly estimations after 13 months was small. However, immunoglobulin concentrations in those children followed for longer than 13 months remained

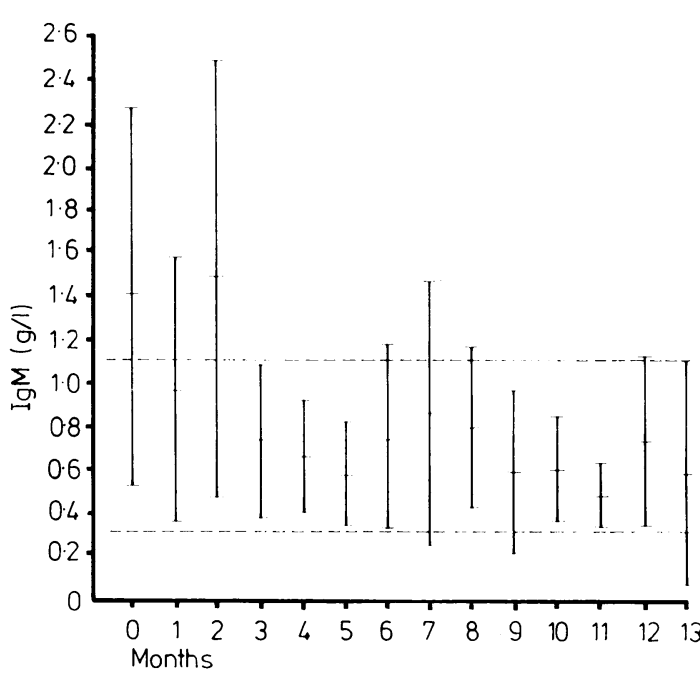

3

Figs 1, 2, 3 Mean concentrations $( \pm S D$ ) of $I g G$, $\operatorname{IgA}$, and IgM at monthly intervals after diagnosis. The broken lines show the normal range of Stiehm and Fudenberg ${ }^{2}$ for comparison.

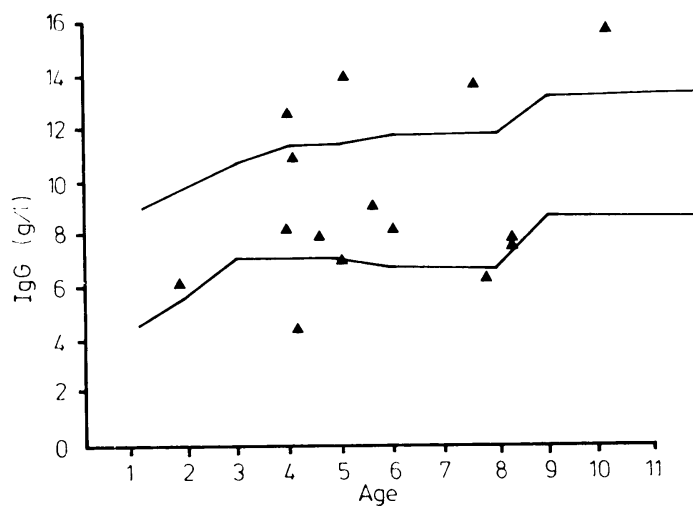

Fig. 4 IgG concentrations of individual children at diagnosis. The lines show the normal range for age of Stiehm and Fudenberg ${ }^{2}$ for comparison.

relatively stable and showed no tendency to rise. T) In Figs 4, 5, and 6, IgG at diagnosis and three months and six months after diagnosis are shown foo each child from whom a specimen was obtained an\& compared with Stiehm and Fudenberg's ${ }^{2}$ normap range for age.

\section{Discussion}

There is wide variation in published reports of immunoglobulin concentrations in children wit 


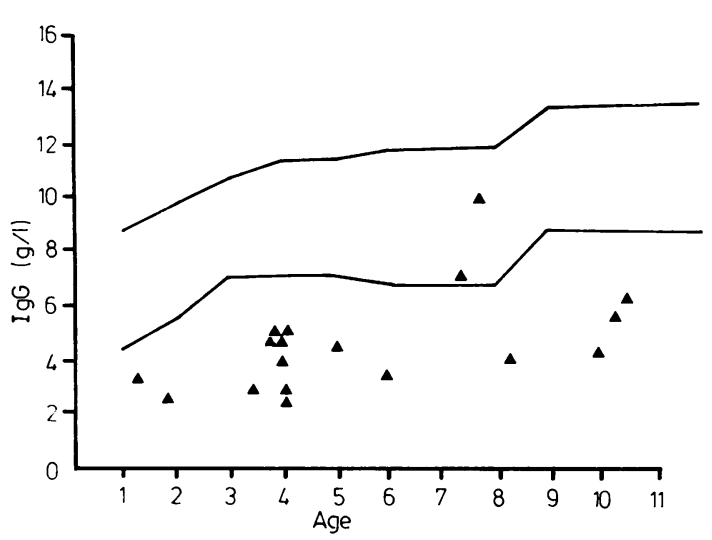

Fig. 5 IgG concentrations of individual children three months after diagnosis.

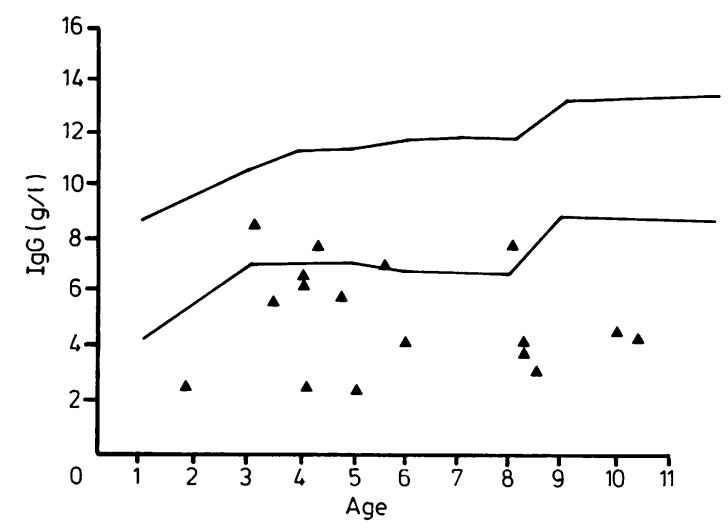

Fig. 6 IgG concentrations of individual children six months after diagnosis.

ALL. Normal levels have been reported at diagnosis by several workers. ${ }^{3-6}$ Others have found depressed $\mathrm{IgG}^{7}$ and depressed $\mathrm{IgA}^{8}$ and depressed concentrations of all three major classes of immunoglobulins. ${ }^{9}$ This study does not suggest depressed concentrations of immunoglobulins at diagnosis. IgG and IgA were probably normal, but many children had markedly raised IgM possibly reflecting recent stimulation of the humoral arm of the immune system. Some ${ }^{910}$ have associated low IgG at diagnosis with a poor prognosis. In this study the IgG was not depressed. The lowest IgG at diagnosis was $4.4 \mathrm{~g} / \mathrm{l}$. It is not possible to comment on this suggested indicator of prognosis.

Many workers ${ }^{3} 711$ have noted a fall in immunoglobulin concentrations after starting treatment. Kiran and Gross $^{3}$ reported a fall within five weeks of starting treatment. Our results show that within one month of starting induction treatment with vin- cristine and prednisone IgG in particular had already begun to fall. Figs 1-3 suggest that although mean concentrations fall they appear to remain within the normal range. The extent of the fall is, however, masked by the width of the normal range when a group with a wide age range is studied. When individual results of IgG were plotted against age in Figs 4-6, most fell to below normal. This is also true for IgA but not for IgM. The fact that IgG began to fall within one month of diagnosis strongly suggests that vincristine and prednisone may be responsible. The half-life of IgG is about 25 days. Therefore the rate of fall is consistent with the humoral arm of the immune system having received an insult immediately after diagnosis. The finding of normal IgG at diagnosis suggests that the leukaemia process itself did not initiate the fall. Similar arguments may be applied to the fall in $\operatorname{IgA}$ and IgM, which have an even shorter half-life. It is of interest that the timing of this fall in IgG and IgA is different from that of peripheral blood B lymphocytes, most of which are IgM-bearing cells. These cells are probably depleted by prophylactic cranial irradiation given between five and nine weeks of diagnosis. ${ }^{1}$ Induction chemotherapy may affect preferentially those $B$ lymphocytes that are in the process of maturing into IgG- or IgA-secreting plasma cells.

In most published series immunoglobulin concentrations have risen towards normal during maintenance treatment. In this study the children received continuous 6-mercaptopurine and weekly methotrexate as maintenance treatment, but in addition intermittent pulses of vincristine and prednisone were given monthly for the first six months and then at three-monthly intervals. The trend for immunoglobulin concentrations to fall stops about six months after diagnosis. Thereafter only IgG concentrations show a tendency to rise but never recover completely to pretreatment concentrations. This may reflect the use of frequent reinductions of vincristine and prednisone. It is likely that suppression of the humoral arm of the immune system persists in some degree throughout treatment. Borella and Webster ${ }^{12}$ found depressed IgG in a quarter of the children they studied between 8 and 28 months after diagnosis. After stopping treatment a spontaneous rise in IgM and IgG occurred and a "rebound" in the numbers of bone marrow Igbearing lymphocytes has been described. ${ }^{13}$

Continuous maintenance treatment may be more immunosuppressive than intermittent treatment. ${ }^{14}$ However, when the humoral arm of the immune system was examined by Garay et al.,15 no difference was found in the degree of depression of IgM after continuous or intermittent treatment. In addition 
there was no depression of $\operatorname{IgG}$ or $\operatorname{IgA}$ after either treatment. More recently Rapson et al. ${ }^{16}$ reported immunoglobulin concentrations in three groups of children receiving "continuous," "intermediate," or "intermittent" maintenance regimens. Our course of treatment resembled most closely their "continuous" regimen in which they also found that only IgG showed a tendency, albeit slight, to rise with treatment. It was not possible, however, to see whether the induction period itself caused significant changes in immunoglobulin concentrations.

Our study suggests that the fall in IgG and $\operatorname{IgA}$ at least and the partial recovery of IgG levels may be related to the variation in timing of vincristine and prednisone pulses rather than to continuous administration of 6-mercaptopurine and methotrexate. The pattern of fall of IgM leaves open the possibility that cranial irradiation, continuous maintenance treatment, or a combination of these may be the predominant initiating factor.

We thank Professor W Walker and Dr TC Noble who allowed us to study their patients and the Tyneside Leukaemia Research Association who supported this work.

\section{References}

${ }^{1}$ Reid MM, Craft AW, Todd JA. Serial studies of numbers of circulating $\mathrm{T}$ and $\mathrm{B}$ lymphocytes in children with acute lymphoblastic leukaemia. Arch Dis Child 1977;52: 245-7.

${ }^{2}$ Stiehm ER, Fudenberg HH. Serum levels of immunoglobulins in health and disease: a survey. Pediatrics 1966;27:715-27.

${ }^{3}$ Kiran O, Gross S. The G-immunoglobulins in acute leukemia in children. Hematologic and immunologic relationships. Blood 1969;33:198-206.

4 Ragab AH, Lindqvist KJ, Vietti TJ, Choi SC, Osterland CK. Immunoglobulin pattern in childhood leukemia. Cancer 1970;26:890-4.

${ }^{5}$ Gooch WM, Fernbach DJ. Immunoglobulins during the course of acute leukemia in children. Effects of various clinical factors. Cancer Res $1971 ; 25: 997-1001$.

${ }^{6}$ Hitzig WH, Plüss HJ, Joller P, Pilgrim U, Tacier-Eugster $\mathrm{H}$, Jakob M. Studies on the immune status of childrenos with acute lymphoblastic leukemia, I. Clin ExpO Immunol 1976;26:403-13.

${ }^{7}$ Chandra RK. Serum immunoglobulin levels in children $\frac{\bar{C}}{\bar{C}}$ with acute lymphoblastic leukaemia and their mothers and sibs. Arch Dis Child 1972;47:618-20.

${ }^{8}$ McKelvey E, Carbone PP. Serum immunoglobulin concentrations in acute leukemia during intensives chemotherapy. Cancer 1965;18:1292-6.

${ }^{9}$ Khalifa AS, Take H, Cejka J, Zeulzer WW. Immunoglobulins in acute leukemia in children. $J$ Pediatr 1974;85:788-91.

${ }^{10}$ Haghbin M, Murphy ML, Tan CC, et al. A long-ternin clinical follow-up of children with acute lymphoblastic leukemia treated with intensive chemotherapy regi- $\underset{+}{+}$ mens. Cancer 1980;46:241-52.

${ }^{11}$ Hersh EM, Carbone PP, Wong VC, Freireich EJ. Inhibition of the primary immune response in man by anti- $\sigma$ metabolites. Cancer Res 1965;25:997-1001.

12 Borella L, Webster RG. The immunosuppressive effects of long-term combination chemotherapy in children withacute leukemia in remission. Cancer Res $1971 ; 31: 420-6$. $>$

${ }^{13}$ Sen L, Borella L. Immunological rebound after cessation of longterm chemotherapy in acute lymphoblastic leukaemia: changes in $T$ and $B$ cell populations in bones

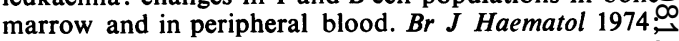
27:477-87.

${ }^{14}$ MacLennan ICM, Kay HEM, Festenstein M, Smith PS Analysis of treatment in childhood leukaemia II. Timing and toxicity of combined 6-mercaptopurine and metho trexate maintenance therapy. Br J Haematol 1976;33 179-88.

15 Garay CE, Pavolovsky S, Sasain M del C, Pizzolato MA $\stackrel{\mathbb{Q}}{\Omega}$ Binsztein N, Eppinger-Helft $M$. Immunocompetence and prognosis in children with acute lymphoblastic leukemia; combination of two different maintenance therapies. Med Pediatr Oncol 1976;2:403-15.

${ }^{16}$ Rapson NT, Cornbleet MA, Chessells JM, Bennett AJ. Hardisty RM. Immunosuppression and serious infection: in children with acute lymphoblastic leukaemia: comparison of three chemotherapy regimes. $\mathrm{Br}$ Haematol 1980;45:41-52.

Requests for reprints to: Dr MM Reid, Department of Haematology, Royal Victoria Infirmary, Newcastle upon Tyne, England. 